\title{
Hypopharyngeal Carcinoma by AJCC v7 Stage
}

National Cancer Institute

\section{Source}

National Cancer Institute. Hypopharyngeal Carcinoma by A/CC v7 Stage. NCI Thesaurus.

Code C91251.

A term that refers to the staging of hypopharyngeal carcinoma according to the American Joint Committee on Cancer, 7th edition. 